\title{
THE ANXIETY OF INFLUENCE: UNA REVISIÓN DE LA POÉTICA DE LUIS CERNUDA A TRAVÉS DEL MITO DE NARCISO
}

\author{
EVA MARÍA RoMERo RIVERO \\ Unidad de Programas Educativos de Badajoz. \\ Consejería de Educación y Empleo (Extremadura)
}

Resumen

Este trabajo se propone llevar a cabo un recorrido por la presencia de la historia de Narciso en la obra de Luis Cernuda, permitiendo apreciar la evolución y el tratamiento que el autor sevillano confiere al mito, todo ello desde una dimensión diacrónica, a la luz de la revisión del concepto de tradición, pero partiendo de las teorías de H. Bloom acerca de la noción anxiety of influence, junto con las ideas de T. S. Eliot contenidas en los ensayos «The Tradition and the Individual Talent» $y$ «What Is a Classic?» y las de E. Said, de forma complementaria, en relación al concepto de admiration for predecesors.

Palabras clave: Generación del 27, Luis Cernuda, Mitología clásica, tradición, influencia.

\section{THE ANXIETY OF INFLUENCE: A REVIEW OF LUIS CERNUDA'S POETRY THROUGH THE MYTH OF NARCISSUS}

\begin{abstract}
The aim of this paper is to examine the presence of the story of Narcissus in the work of Luis Cernuda, allowing us to appreciate how the Sevillian author develops and treats the myth, all of this from a diachronic dimension and from the perspective of the revised concept of tradition, but starting from the theories of $\mathrm{H}$. Bloom concerning the notion of 'anxiety of influence', together with. The ideas of T. S. Eliot contained in the essays «The Tradition and the Individual Talent» and "What Is a Classic?», as well as those of E. Said, in a complementary way, in relation to the concept of 'admiration for predecessors'.

Keywords: Generation of '27, Luis Cernuda, classical mythology, tradition, influence.
\end{abstract}




\section{INTRODUCCIÓN}

Explica Bloom que la obra de los grandes poetas es una consecuencia de la disputa que éstos mantienen con sus antecesores, con el objetivo de alcanzar una voz plena e individual, es decir, mantienen una lucha, en principio, con la tradición, ya que, según el crítico americano, «los poetas fuertes forjan esa historia malinterpretándose unos a otros para despejar un espacio imaginativo para sí mismos» (Bloom, 2009: 55). Tanto es así, que lo que lleva al poeta a esa lucha es la angustia de ser un epígono prescindible, que tratará de afirmarse mediante la negación de la tradición poética, lo cual, no deja de ser una relectura en la que se establecen inevitablemente relaciones intertextuales (Bloom, 2009: 25-26). Por otra parte, T.S. Eliot basa su definición de lo clásico en la madurez y en la universalidad, ligando el concepto de tradición al de originalidad (individual talent), es decir, la tradición se actualiza gracias al genio del poeta, que la reestructura estableciendo un diálogo cuyo resultado sería una concepción global de la creación (Eliot, 2004: 19).

En esta encrucijada creativa se sitúa el mito de Narciso en la obra de Cernuda. El poeta parte de la tradición clásica para tratar de encontrar su lugar en la literatura -y el mundo- mediante la recreación de mitos clásicos, o loci comunes, presentes en la colectividad europea. De esta manera, el sevillano emplea la tradición, modulándola en función de su experiencia personal e íntima, introduciendo nuevos elementos, extraídos de su propia situación vital, circunstancia sin la cual no puede entenderse su obra, pues "pocas veces, como en el caso de Luis Cernuda, la trayectoria poética va tan íntimamente ligada a la aventura personal del autor» (Talens, 1975: 53). Esto nos permite apreciar esa integración que mencionábamos antes, pero como signo de «superación frente a los predecesores» (Said, 2004), como demuestra el siguiente poema en prosa de Ocnos, «El poeta y los mitos» (la cursiva marcada es mía):

Bien temprano en la vida, antes que leyeses versos algunos, cayó en tus manos un libro de mitología. Aquellas páginas te revelaron un mundo donde la poesía, vivificándolo como la llama al leño, transmutaba lo real. Qué triste te pareció entonces tu propia religión. Tú no discutías ésta, no la ponías en duda, cosa difícil para un niño; mas en tus creencias hondas y arraigadas se insinuó, sino una objeción racional, el presentimiento de una alegría ausente. ¿Por qué se te enseñaba a doblegar la cabeza ante el 
sufrimiento divinizado, cuando en otro tiempo los hombres fueron tan felices como para adorar, en su plenitud trágica, la hermosura?

Que tú no comprendieras entonces la casualidad profunda que une ciertos mitos con ciertas formas intemporales de la vida, poco importa; cualquier aspiración que haya en ti hacia la poesía, aquellos mitos helénicos fueron quienes la provocaron y la orientaron. Aunque al lado no tuvieses alguien para advertirte del riesgo que así corrías, guiando la vida, instintivamente, conforme a una realidad invisible para la mayoría, y a la nostalgia de una armonía espiritual y corpórea rota y desterrada siglos atrás de entre las gentes.

Pese a esto, Cernuda va más allá, hasta el punto de que Narciso es clave para entender su poesía, tal como ya han destacado algunos de sus más canónicos estudiosos (Derek Harris, Cristina Mabrey o Martínez Nadal); sin embargo, desde el presente estudio pretendemos sugerir otras combinaciones hermeneúticas, máxime teniendo en cuenta que, para este autor, la creación literaria transita por este mito al suponer una historia que, no solo le obsesiona, sino que representa su condición homosexual, en tanto que consolida su propio universo artístico, arraigado, indistintamente, en una profunda soledad (Gómez-Castellano, 2018: 367368). Así, su forma de afrontar el anxiety of influence supondría entender que el concepto de originalidad en la obra del poeta sevillano está presente, no como negación y posterior reafirmación, sino como recreación, adaptación e integración. Cernuda, lejos de distanciarse, incluye la tradición en su propia experiencia vital, la admiración por los predecesores se hace patente asimilándola a esa lucha perpetua que los poetas mantienen con otros poetas anteriores (Bloom 2009: 73).

Es indudable el hecho de que Narciso es una de las historias mitológicas más transitadas por el arte y la literatura española y universal de todas las épocas (Alganza Roldán, 2010), si bien es cierto que, con la «difusión de la obra de Sigmund Freud Introducción al narcisismo, de 1914, el mito recobra centralidad en el Modernismo y la Generación del 27» (Bianchi, 2019: 559-560). De ahí que Cernuda no sea el único autor de la Generación de 27 que lo utiliza. También podemos encontrarlo en Alberti, Federico García Lorca, Aleixandre, etc., dejando una estela que retomarán después algunos autores de los cincuenta (Bianchi, 2019: 564565). Sin embargo, para el poeta sevillano el anxiety of influence por los 
clásicos en general, así como por el mito de Narciso y sus modulaciones en particular, se convierten en un tema recurrente y casi «marca de autor», pues, como afirma Alganza Roldán (2010: 187), «la figura de Narciso sirve a Cernuda para formular esa verdad íntima que vertebrará su poesía», marcada por las circunstancias existenciales a las que está unida y por la personalidad del poeta, que lo distancian del tratamiento ofrecido por otros miembros de su generación, de tono más burlesco o pasado por el molde del neopopularismo (Román, 2003: 394-395).

El corpus aquí seleccionado trata de presentar un sucinto análisis de la importancia que Narciso tuvo en la obra de Cernuda bajo el prisma de los estudios de Bloom y Eliot mencionados más arriba, por lo que aparecen recogidos textos de prácticamente todos sus libros organizados según las sucesivas etapas que el autor va superando.

\section{ELEMENTOS Y CARACTERIZACIÓN DEL MITO}

Existen varias versiones del mito de Narciso; la más conocida es la de Ovidio, plasmada en las Metamorfosis, según la cual Narciso es hijo de Cefiso y la ninfa Liríope. Al nacer, el adivino Tiresias vaticina que el niño vivirá hasta viejo si no se contempla a sí mismo. Con la edad adulta numerosas ninfas lo pretenden, pero él las desprecia a todas. Cuando la ninfa Eco se enamora de él y Narciso la rechaza, desesperada, se marcha a un lugar apartado donde adelgaza tanto que lo único que queda de ella es su voz quejumbrosa. Ante esto, la diosa Némesis hace que el joven se incline sobre una fuente para calmar su sed. Allí contempla su rostro reflejado, del que se enamora, dejándose morir inclinado sobre su propia imagen. En el lugar de su muerte creció una flor a la que se le dio su nombre.

El relato trata de ser la explicación del nacimiento de la flor del narciso y su ubicación siempre próxima a los ríos, aunque no debemos olvidar la primera parte del mito que dilucida el origen del fenómeno del eco. Estamos, por lo tanto, ante una historia de amor. Sin embargo, el mito ha pasado a la lengua como una expresión lexicalizada con la que se describe a alguien petulante, presumido, omitiendo el factor amoroso de la ninfa y quedando únicamente el componente del amor reflexivo con el 
nuevo giro de la preocupación por el aspecto propio, característica con que se dota a un «narciso» actual.

Los motivos principales por los que podemos reconocer la historia de Narciso en la literatura son dos: la superficie reflectante, que puede o no ser el agua, y el final trágico, no necesariamente la muerte. Cernuda, en su reelaboración personal, incluirá otros elementos que nos permitan, en muchos casos, identificar determinadas referencias en los poemas al tema que nos ocupa, dándole una modernidad y un tratamiento propio muy original. En este sentido, la coyuntura vital del poeta hace que la historia de Narciso sea un mito fácilmente asimilable a sus sentimientos, pues, partiendo de su homosexualidad, eje del mundo interior de Cernuda, y de unas condiciones de soledad especialmente dolientes para el andaluz, que, finalmente, le conducirán a un autoerotismo en el que se «equipara simbólicamente el acto de escribir poesía y el acto de estimularse eróticamente en solitario» (Gómez-Castellano, 2018: 362-63) se modulará, como veremos, el mito de Narciso en la lírica cernudiana.

La concepción del amor para Cernuda tiene ascendencia neoplatónica, «es un sentimiento diferente al deseo», según el cual «la aspiración frustrada del hombre será buscar la otra mitad que lo complete» (Ramos Ortega, 1982: 138); así, este sentimiento supondrá un medio para integrarse en su otra mitad. Esto lo expresa muy bien el autor en el poema "Propiedades» de Variaciones sobre tema mejicano: "Cuando tenemos afecto a una criatura queremos ser como esa criatura, queremos ser esa criatura». Para plasmar ese amor, Cernuda pone a su servicio la mitología clásica, como nos demuestra muy bien el poema ya mencionado «El poeta y sus mitos» de Ocnos. La relación entre este concepto del amor cernudiano y el mito de Narciso puede muy bien resumirse, en palabras de Ramos Ortega (1982: 139), como «la analogía entre el ser incompleto del ego y el hecho de que Narciso sea en realidad dos personas. Lo que ocurre es que Narciso, al igual que Cernuda, nunca podrá hacerse uno con su ser reflejado».

Podemos fechar hacia 1925 el comienzo de la actividad literaria de Cernuda, adscrito por esos años a la vanguardia estética de la poesía pura, movimiento tutelado por Juan Ramón Jiménez y basado en los análisis orteguianos de La deshumanización del arte, los ideales geométricos del cubismo y el esteticismo de Valéry. Su primer libro, Perfil del aire, es 
la culminación de Cernuda como poeta puro y aún los cuatro poemas de la serie Égloga, elegía, oda están en la órbita de la poesía purista, de ahí que la hayamos comprendido en esta primera etapa. Hemos incluido también la prosa El indolente, por el interés en observar el tema de Narciso y su proyección en toda la producción literaria de Cernuda.

La décima «Se goza en sueño encantado» es clave, no solo por ser la primera aparición, de manera explícita -cosa que no volverá a suceder en ningún texto-, del mito de Narciso, sino porque es una composición en la cual no se produce identificación. Podríamos decir que el poema se dedica exclusivamente a narrar la anécdota mitológica con un lenguaje y estilo muy gongorino, en especial, en lo referente a las metáforas («diamante azogado», «humanas rosas»), aunque ya aparecen esbozados los elementos representativos y aquellos que después utilizará Cernuda en las posteriores reformulaciones del mito, como, por ejemplo, el sueño. Esta idea, que será medular en las composiciones cernudianas, muestra con frecuencia el motivo del reflejo del otro, bajo el tamiz de la visio o la ensoñación, por el que nos lleva igualmente a semas de embelesamiento, indolencia, etc. Ejemplos como «se goza en sueño encantado»o «hurta a sus ojos ausente» entroncan con la situación genérica de muchos poemas donde se trata este mito: el joven adolescente que contempla a través de una ventana o un espejo. Igualmente, los sonetos «Vidrio de agua en mano del hastío» y "La desierta belleza sin oriente» evocan sentimientos de indolencia basados en una experiencia narcisista; así, en el primer soneto de los mencionados, el adolescente busca consuelo en la poesía: «La soledad, tras las puertas cerradas, / abre la luz sobre el papel vacío». Si bien se da cuenta de que ésta solo puede ofrecer una imagen falsa; es entonces cuando se sumerge en sus propios sueños de manera narcisista, pero con esa indolencia característica de estos poemas de la que antes hablábamos: «El sueño, embelesado e indolente». Del mismo modo, en «La desierta belleza sin oriente» los rasgos de embelesamiento son aún más evidentes; el adolescente es reflejo de sí mismo en el espejo, pero es un reflejo fingido, falso, que se dirige hacia un imposible objeto amoroso: "Y el tiempo mira a un cuerpo que se sueña / en el cristal, fingido irreparable». El espejo se convierte por tanto en un símbolo de otredad, de manera que «el poeta se mira en el espejo de su arte y el lector se mira en la representación artística del poeta» (Castro, 2005: 185). 
Diferente innovación en el tratamiento del mito es la imagen que tiene el andaluz de Narciso como un ser solitario, característica que podemos entender a la perfección si la suscribimos a la personalidad del propio poeta y a lo mencionado más arriba:

Luis Cernuda [...] construyó una filosofía estética que tenía en su centro una noción muy compleja de la masturbación como metáfora de la soledad del artista homosexual y como símil poético de la creación estética, en este caso de poesía (Gómez-Castellano, 2018: 372).

Aunque esta soledad, de índole amorosa, no es buscada, como sí podía parecernos en la narración mitológica original, pues Narciso rechaza a todas las ninfas y en especial a Eco; sin embargo, en Cernuda esta soledad es forzada, como una condena o un castigo: «Soledad sin amor ni claro día».

Siguiendo con los elementos representativos del mito, nos ocuparemos del agua, que Cernuda modula en esta primera etapa como el cristal, a través de la analogía hecha entre aquella y la superficie reflectante, debido al tono interiorista que tienen las composiciones y a la situación espacial en que la se desarrollan (la habitación del adolescente): «En donde la luz se irisa / tornasol, sino el cristal» o «En el cristal, fingido irreparable» o incluso en «Vidrio de agua en mano del hastío».

Tan solo la décima «Se goza en sueño encantado» emplea el elemento original del mito, pues, como ya dijimos, esta composición es una recreación del relato clásico. El texto más claro donde puede observarse lo dicho es quizá «El indolente», que refiere una honda experiencia narcisista reflejando la aguda autoconciencia que Cernuda tiene, ya en esos años, de sí mismo. Manifiesta, además, al igual que en las otras composiciones, la expresión de soledad e inseguridad del propio poeta: «al separarme del cristal me pierdo a mí mismo».

En el poema Égloga, también de esta etapa, el ambiente es distinto de los poemas anteriores; se localiza en exterior, un «idílico paraje» que casi podríamos llamar locus amoenus, dotado además de una serie de alusiones mitológicas que casan a la perfección con el paisaje descrito. Se alude a una «flauta impura», a «unas cañas», a «unas ninfas verdaderas», todo ello en un paisaje "nativamente digno de dioses», que nos remite de inmediato a la historia de Pan y la siringa, relacionado a su vez con el mito 
de Eco, como recoge la novela de Longo, Dafnis y Cloe, III, 21-23. Existen, de hecho, referencias al "eco», esa parte olvidada del mito de Narciso, aunque no podemos afirmar que sea así con los datos que nos proporciona el texto.

El personaje, de nuevo un adolescente solitario, muere al verse reflejado en el agua, como el Narciso mitológico. El poema, no obstante, está plagado de semas de muerte; el final trágico del mito se cumple. Quizá la nota más característica de estas connotaciones trágicas sean las alusiones al agua congelada, estilema de muerte en Cernuda: "Sus hielos liquida» o "De congeladas, pálidas espumas» que podemos ver en otros poemas como el ya citado «Se goza en sueño encantado» ( $\mathrm{O}$ agua helada»). Por lo demás, esta composición posee prácticamente todos los elementos con que el poeta andaluz modula el mito (soledad, belleza, agua, etc.) y que seguiremos viendo en otras etapas.

Pertenecen a la época más surrealista los textos de Los placeres prohibidos, «un libro de amor, de rebelión y de belleza» (Cernuda, 1999: 19) en el que podemos observar plenamente la cosmovisión de Cernuda, que proclama la libertad del cuerpo, el goce del amor y su soledad y el deseo -máxima cernudiana- como la vibración suprema de la vida.

En Los placeres prohibidos vemos además una experimentación formal, la mezcla de poesía en verso y en prosa, que sabiamente culminará en Ocnos y Variaciones sobre tema mexicano. De esta forma y como muestra, se adecua al tema un bello poema en prosa, "Estaba tendido», encuadrado dentro del subgénero de narraciones oníricas, tan usuales en el Surrealismo y que Cernuda cultiva con frecuencia en este libro. El punto de partida es el agua, elemento clave a partir de ahora en todas las composiciones. Es un relato en primera persona en el que el sujeto lírico tiene entre sus brazos un cuerpo con el que solo es posible tener contacto físico gracias al agua: «Lo besé en los labios, porque el río pasaba por debajo» $\mathrm{O}$ «Lo besé en las espaldas porque el agua sonaba por debajo de nosotros».

Esto ya nos hace pensar en la posibilidad de que este cuerpo sólo exista a través del agua; sería, por tanto, el reflejo del propio yo. Este cuerpo-reflejo se desvanece y el motivo del agua continúa presente como testigo para siempre del dolor causado (puñal), el amor (ala) y el recuerdo (sombra), que persigue al yo. 
Identifico el amor (también el deseo) con la imagen del ala porque la única nota física que se nos da de ese amante ideal es la espalda, símbolo de la sensualidad, que es como «dos alas plegadas», que nos llevarían en un primer momento a reconocer un ángel o una criatura celestial; sin embargo, dado el contexto amoroso del poema, es más lógico pensar en el dios alado del amor, Cupido. Cabe señalar que este poema tiene una estructuración perfecta basada en estructuras bimembres y, aunque formalmente aparezca como prosa, posee todas las demás características del género lírico.

El otro poema de este libro «Veía sentado», que, por cierto, tiene un título en parte paralelo con el anterior (verbo en pretérito imperfecto, solo que aquí transitivo y allí intransitivo, y participio). Se estructura bajo la anáfora del verbo de percepción "veía», que reivindica de forma clara la identidad homosexual a través de «la relación entre lo verbal y lo visual» (Castro, 2005: 185) llevándonos, en primer término, por una descripción situacional, un paisaje al aire libre, que contrasta con los interiores de la primera época, para pasar a una reflexión metafísica, en lo que supone un esquema composicional muy clásico ${ }^{1}$. La novedad de esta obra es que el yo no muere al entrar en contacto con el cuerpo que se refleja en el agua, sino que éste se funde con el sujeto. Esto ocurre por el carácter existencial del poema; el reflejo no es como en otros poemas expresión de soledad, sino de autoconocimiento, de ahí la metáfora que establece Cernuda del agua como verdad, algo habitual en el poeta. En otros poemas, no obstante, el agua es el amor, la música, el símbolo del pasado, etc. "Veía al inclinarme sobre la verdad».

Con Donde habite el olvido Cernuda comienza a interesarse por el Romanticismo, en particular el nórdico, que le lleva -paradójicamentea conectar con Grecia y su cultura en una sed de paganismo que une la moral libre y la estética simbolista. Prueba de ello son las traducciones que realiza en esos años, especialmente la de Hölderlin, que publica en Cruz y Raya (noviembre de 1935), en cuya nota introductoria refiere su atracción por el mundo helénico (Cernuda, 1999: 22):

\footnotetext{
${ }^{1}$ Horacio, en la mayoría de sus Odas, empieza con una nota paisajística para desembocar en una reflexión existencial.
} 
[los mitos griegos son] eje de una vida perdida entre el mundo moderno, y para quien las fuerzas secretas de la tierra son las solas realidades lejos de estas otras convencionales por las que se rige la sociedad.

El poema seleccionado es uno de los más llamativos del tratamiento del mito, pues el yo lírico se corresponde en la primera parte con la historia de Narciso y en la segunda con la de la ninfa Eco. El elemento del agua, esencial siempre, aparece en un símil con el amor, fundido a la vez con el yo, de manera que se produce una curiosa mise en abyme.

El cambio de tono lo observamos en un verso clave: «Me he olvidado a mí mismo en sus ondas», que supone el fin de Narciso, pues las ondas se refieren al agua; ahora bien, más arriba se había producido una metáfora comparativa tipo A como B, es decir, «el amor como un agua», con lo cual, las ondas en las que se ha perdido el sujeto lírico son las del amor. Esta situación y los versos que siguen («Vacío el cuerpo»...) nos remiten de inmediato a la parte de la historia de Eco, a la que, recordemos, el amor por Narciso la va anulando poco a poco hasta quedarse en un hilo de voz. Este proceso lo entendemos a la perfección en el verso nueve, en el que sentencia "Soy eco de algo». El final de la composición sorprende por lo sentencioso: es una conclusión dual que parece afirmarse con el desdoblamiento múltiple planteado en el poema.

Cernuda inicia con Las nubes una nueva etapa que podríamos llamar de madurez, en la que entrará con más fuerza que en las obras anteriores la experiencia cultural, biográfica e histórica. La poesía del sevillano cambia de tono, se vuelve más objetiva, aunque, a medida que es más él, utiliza más técnicas de distanciamiento para procurar esa sensación de objetividad mencionada.

Dentro de esta etapa se incluye el libro Como quien espera el alba, una obra llena de introspección, «el libro más clásico de Cernuda entendiendo por ello no el mejor, naturalmente, sino el más equilibrado» (Cernuda, 1999: 36). El poema "Vereda del cuco» se incluye en esta obra y supone un extenso texto de reflexión, donde se presenta el mito de Narciso transitado por las diferentes etapas vitales. El eje es el agua, en concreto, una fuente a la que el sujeto lírico acude primeramente de niño «a conocer tu sed aún inexperta» y donde ya se produce la apariencia del otro en el agua. En la edad madura, de nuevo hay un acercamiento a la fuente, esta vez "para alcanzar la luz», metáfora del conocimiento y que 
en Cernuda sería más bien del autoconocimiento a través del amor. Ahora el amor le hiere, pero no puede alejarse de ese camino: «Y entonces no pudiste / desertar la vereda / oscura de la fuente»; pese a que sabe que el final es la muerte: "Aunque no puede el labio / beber dos veces de la misma agua» o más adelante "Voz pura es la palabra porque suena en esa fuente, / y la muerte es de ella el fondo codiciable».

El juego de desdoblamiento en este poema viene dado principalmente por el uso de la segunda persona gramatical, el tú, que hace pensar en un interlocutor y, no es más que un alter ego del propio autor. Este procedimiento especular será característico sobre todo del último Cernuda, que lo utiliza para distanciarse, especialmente en poemas que aluden a experiencias vitales más o menos reconocibles y que el poeta interioriza (por ejemplo, en Ocnos y en Variaciones sobre tema mexicano). Por otro lado, resulta muy acertado para el tratamiento del mito, pues Narciso se refleja en el agua, al igual que Luis Cernuda en su obra.

Es evidente, por lo que venimos analizando, que el elemento fundamental en la configuración cernudiana del mito de Narciso es el agua, aspecto por el que reconocemos el mito en primer término; no obstante, una lectura atenta nos revela que están presentes otros motivos que caracterizan el mito en el poeta sevillano. En primer lugar, encontramos algunos semas de ensimismamiento, tan característicos de la primera época: "Mirando con asombro mudo» o "Estático en su orilla». Asimismo, se presenta de manera recurrente el motivo del beso y los labios, que marcan el final de ese reflejo. En el poema, mientras el niño aún no ha intentado besar la imagen, las visitas a la fuente son posibles, pero, cuando este hecho se produce, el yo sabe que es el final, que nunca podrá besar esa imagen, aunque vendrán otras, ya que «el amor es lo eterno y no lo amado». La soledad, otro de los componentes esenciales del mito, está en el ambiente de esta poesía; no son necesarios datos concretos, por ser algo patente en la propia anécdota del poema; el sujeto lírico baja a esa fuente sin compañía y, ya mayor, contempla a los jóvenes como un voyeur (hecho real por el que fue muy atacado por algunos colegas y contemporáneos, como Juan Ramón Jiménez). A este sentido de soledad siempre presente se refiere el poeta en una de sus composiciones de Ocnos: «La soledad está en todo para ti y todo para ti está en la soledad». 
Por su parte, el poema «Vereda del cuco» termina con una referencia mitológica que nada tiene que ver con Narciso, pero que no sorprende porque se asocia al tópico de la flamma amoris, que recorre todo el poema, el amor visto como un ave fénix que cíclicamente muere y renace de sus cenizas.

En otro orden de ideas, la obra Vivir sin estar viviendo refleja desde el título el decaimiento del autor: el exilio, el clima londinense y un especial momento crítico hacen que este libro no suponga un gran avance respecto a los planteamientos de Como quien espera el alba. Dentro de éste, "El intruso» es un poema, como el anterior, de juegos especulares con la segunda persona. Supone una mirada hacia el pasado y una identificación con ese pasado, aunque resulta curioso que un poema de esta fecha tome como elemento reflectante de nuevo el espejo, pues ya vimos que era característico de la primera etapa de Perfil del aire. No obstante, otros elementos como la ensoñación y la soledad aparecen igualmente formuladas, con lo que nos ayudan a reconocer el mito más allá del elemento acuático: «En vano ante el umbral de un sueño».

El encuentro con su lengua, el clima y un temperamento parecido al de su país natal harán despertar en el sevillano su sensibilidad dormida, poniendo en marcha nuevos mecanismos de la expresión poética. Buena prueba de esta experiencia será el libro Variaciones sobre tema mexicano, pero también el genial Con las horas contadas y sobre todo Desolación de la quimera.

«Luis de Baviera escucha Lohengrin» es una excepcional muestra de la altura del libro en que está incluido. Es un poema donde confluyen no sólo el mito de Narciso, sino también una serie de figuras de la mitología nórdica (elfos), todo ello pasado por otra arte, la música, que aquí cobra especial importancia debido a la asimilación que se produce con el agua, del mismo modo que el mito del fauno Pan y su flauta de pastor aparecían fundidos con el mito de la musical ninfa Eco y de Narciso en la mitología grecolatina. El personaje es una extraña figura, un elfo, que asiste misteriosamente a una representación de la ópera de Wagner, Lohengrin, y que supone un desdoblamiento del propio Cernuda a través de un interesante juego especular; el príncipe se desdobla en el elfo y este príncipe, a su vez, es alter ego de Cernuda, que en un momento de la composición se desdobla nuevamente a través de la figura de Narciso. Se va 
creando de esta manera un ambiente de arrobamiento gracias al cual asistimos al desdoblamiento del personaje. La música es en esta ocasión el elemento reflectante:

Sobre la música inclinado, como extraño contempla

con emoción gemela su imagen desdoblada

y en éxtasis de amor y melodía queda suspenso.

Se inclina y se contempla en la corriente

melodiosa e imagen ajenada, su remedio espera.

Cernuda ya había dado indicios de que esto podía ser así; por ejemplo, en un fragmento de un poema de Ocnos leemos:

Pero a la música hay que aproximarse con mayor pureza, y solo desear en ella lo que ella puede darnos: embeleso contemplativo. En un rincón de la sala, fijos los ojos en un punto luminoso, quedaba absorto escuchándola, tal quien contempla el mar. Su armonioso ir y venir, su centelleo multiforme, eran tal la ola que desalojase las almas de los hombres. Y tal la ola que nos alzara desde la vida a la muerte, era dulce perderse en ella, acunándonos hacia la región última del olvido.

El otro elemento al que hemos aludido como principal en las demás composiciones, el sueño, cobra aquí una importancia clave, con numerosas alusiones directas: «en el palco real un elfo solo asiste» $\mathrm{O}$ «El brotar melodioso que le acuna y nutre / los sueños, mientras la escena se desarrolla». Pero, de nuevo, su importancia radica en el hecho de que mana de la música, cosa que no había sucedido antes: «Flotando sobre música el sueño ahora se encarna» o «es la música quien nutriera a su sueño».

Semas de soledad y de incomprensión aparecen dispersos por el texto, de forma que seguimos viendo, pese a ser un poema filtrado por otra arte, que posee todos los componentes habituales de la modulación cernudiana de Narciso: «Donde la soledad y el sueño le ciñen su única corona».

En el poema hay una conciencia del personaje sobre su destino, que podemos asociar enseguida con el mito de Narciso mediante la interrogación retórica «¿no muere aquel que ve a su doble?». El final de este elfo-rey tiene paralelos manifiestos con el Narciso del mito, se transforma 
«como en flor, en cosa hermosa, inerme, inoperante» y termina «fundido con el mito al contemplarlo».

\section{SÍNTESIS FINAL}

De todo lo dicho podemos extraer una idea básica: el mito de Narciso en Cernuda recorre toda su producción, de tal modo que debemos hablar de este hecho como mito personal para el autor; un mito que el poeta modula con una serie de elementos que aparecen reiteradamente en sus composiciones. El más importante es el agua, o, en su defecto, cualquier otra superficie reflectante (el cristal, por ejemplo), aunque esto es más frecuente en la obra de la primera época. El agua se convierte en metáfora del amor, de la verdad, pero la más interesante es la imagen que se produce con la música, característica del Cernuda más tardío, que nos lleva a la intención del sevillano de alcanzar «la idea de la belleza que está más allá de las cosas» (Caballero de Díaz, 2003: 118), en la perpetua tensión de su obra entre la realidad y el deseo. Una realidad ontológica que pasa por la otredad que ofrece, en este punto, no solo el mito de Narciso, sino el complejo mítico Narciso-Eco-Pan, como vemos, verbigracia, en la novela de Longo Dafnis y Cloe.

Otro de los elementos importantes es la soledad, presente en todas las composiciones de manera más o menos explícita, y que supone la esencia y función principal del mito. Narciso, a través del aspecto de la soledad, supone la identificación del poeta con el joven mítico, aunque Cernuda no se equivoca con su reflejo, como Narciso, puesto que, en realidad, su ser amado no es otro que su propio autoconocimiento dentro del plano cósmico de la creación literaria.

El motivo del sueño, por su parte, relacionado con el embelesamiento, es también importante, pues la historia que se presenta aparece bajo el tamiz de lo onírico, con lo que aquellos adquieren un distanciamiento y un ambiente de irrealidad relacionado con el género de la visio, de ahí la multitud de verbos de percepción que se aprecian en los poemas.

Recurrente resulta la imagen del beso, presente ya en el mito clásico, que Cernuda muestra en ocasiones con la metonimia de los labios. Esto se relaciona con otro motivo, la belleza y la juventud del cuerpo que se 
refleja, que ocurre siempre excepto en «El intruso», donde la figura es «vieja»y «hosca».

Pero todo ello no es más que el afán de Cernuda «de dar voz a la identidad homosexual» (Castro, 2005: 185) mediante la utilización de un mito clásico que trae a su experiencia personal e íntima, en lo que podríamos denominar una dialéctica con la tradición que, sin embargo, lejos de terminar en la redundancia, da como resultados textos originales, superando así the anxiety of influence al introducir nuevos elementos extraídos de su situación vital, con lo que consigue darle una distancia a la expresión subjetiva y llevarlo al terreno de lo metapoético, pues el mito se convierte en un reflejo en el que el autor proyecta su soledad como motor de la enunciación poética. Todo con una modernidad y un tratamiento personal del sentimiento que resulta ser una marca de estilo y que lo convierte en un epígono fundamental, necesario para comprender la lucha que otros autores establecen con la tradición clásica y con el propio hecho de la escritura. Así pues, el concepto de influencia está presente en este autor, sin duda, pero con el objetivo de superar the anxiety of influence que la tradición le genera, dando como resultado una fuerte integración en la propia tradición con la que lucha en un ejercicio continuo de metapoesía.

\section{BIBLIOGRAFÍA CITADA}

ALganZa Roldán, M. (2010): Metamorfosis de Narciso en la cultura occidental. Granada: Universidad de Granada.

BIANCHI, M. (2019): «Narciso en tres poetas españoles del medio siglo: reivindicando el derecho al amor». Boletín de la Real Academia Española, xcix.320, 557-578.

Bloom, H. (2009): La ansiedad de la influencia. Una teoría de la influencia. Trad. J. Alcoriza y A. Lastra. Madrid: Trotta.

CABALlero de Díaz, M. E. (2003): «El mito de Narciso en la poesía de Cernuda». Circe, 8, 111-122.

CASTRO, E. (2005): «Los “Narcisos" del surrealismo español: Luis Cernuda, Salvador Dalí y Federico García Lorca», Letras peninsulares, 18.1, 179-200.

Cernuda, L. (1971): Perfil del aire. Ed. D. Harris. London: Tamesis Books. 
260 Eva María Romero Rivero The anxiety of influence: una revisión de la poética de Luis Cernuda ...

Cernuda, L. (1999): Las Nubes. Desolación de la Quimera. Ed. L. A. de Villena. Madrid: Cátedra.

Cernuda, L. (2002): Poesía completa. Ed. D. Harris y L. Maristany. Madrid: Siruela.

ELIOT, T.S. (2004): Lo clásico y el talento individual. México: UNAM.

GómEZ-CASTELLANO, I. (2018): «Autoerotismo y metapoesía: reflexiones en torno al onanismo en las Primeras poesías de Luis Cernuda», Hispanic Research Journal: Iberian and Latin American Studies, 19.4, 361-381.

Ramos ORTega, M. (1982): La prosa literaria de Luis Cernuda: el libro Ocnos. Sevilla: Diputación de Sevilla.

RomÁn, I. (2003): «Los mitos clásicos en la poesía de Federico García Lorca». Anuario de Estudios Filológicos, 26, 387-405.

SAID, E. (2004): Humanism and Democratic Criticism. New York: Columbia University Press.

TALENS, J. (1975): El espacio y las máscaras, introducción a la lectura de Cernuda. Barcelona: Anagrama.

Eva María RoMERo RIVERo Unidad de Programas Educativos de Badajoz. Consejería de Educación y Empleo (Extremadura) cerezasdelcementerio@gmail.com https://orcid.org/0000-0002-7169-9977 